\title{
Study of the influence of initial-state fluctuations on hydro- dynamic simulations
}

\author{
Marcin Słodkowski ${ }^{1, *}$, Patryk Gawryszewski ${ }^{1}$, and Dominik Setniewski ${ }^{1}$ \\ ${ }^{1}$ Faculty of Physics, Warsaw University of Technology Koszykowa 75 str. 00-662 Warsaw, Poland
}

\begin{abstract}
In this work, we are focusing on assessing the contribution of the initial-state fluctuations of heavy ion collision in the hydrodynamic simulations. We are trying to answer the question of whether the hydrodynamic simulation retains the same level of fluctuation in the final-state as for the initial stage. In another scenario, the hydrodynamic simulations of the fluctuation drowns in the final distribution of expanding matter. For this purpose, we prepared sufficient relativistic hydrodynamic program to study $\mathrm{A}+\mathrm{A}$ interaction which allows analysing initial-state fluctuations in the bulk nuclear matter. For such an assumption, it is better to use high spatial resolution. Therefore, we applied the $(3+1)$ dimensional Cartesian coordinate system. We implemented our program using parallel computing on graphics cards processors - Graphics Processing Unit (GPU). Simulations were carried out with various levels of fluctuation in initial conditions using the average method of events coming from UrQMD models. Energy density distributions were analysed and the contribution of fluctuations in initial conditions was assessed in the hydrodynamic simulation.
\end{abstract}

\section{Introduction}

Relativistic hydrodynamics is used to model strongly interacting matter that can be treated as an ideal liquid at some stage. This type of behavior of nuclear matter has revealed experimental data for ultra-relativistic heavy-ion collisions on RHIC and LHC accelerators. Our approach in relativistic hydrodynamics is the use of the Cartesian (three-dimensional) system with time $(3+1)$ on high resolution numeric lattice, which is necessary to describe the evolution of the nucleus + nucleus collision [1]. These types of simulation conditions allow testing sources of perturbation in hydrodynamic observables. Interesting sources of anisotropy are initial-state fluctuations evolution of the $\mathrm{A}+\mathrm{A}$ collision in the pre-equilibrium state, which is the initial conditions of the simulation. Furthermore, it can study the influence of jets in the bulk nuclear matter $[2,3]$. This will allow you to better understand the property nuclear matter under extreme density conditions and separate the collective effects (flow) from those associated with the dynamics of jets (non-flow) measured by elliptic flow $v_{2}$ (a quantitative measure of anisotropy A+A collision reaction [4]).

\footnotetext{
*e-mail: marcin.slodkowski@pw.edu.pl
} 


\section{Hydrodynamics simulations}

\subsection{Hydrodynamics software}

The hydrodynamics software can simulate the collective behaviors in the A+A interactions. Our program is a component of the simulation chain, which contains modules for generating initial conditions, solving differential equations, checking freeze-out, calculating hypersurface using Cooper-Frye sampler. The main component solves the set of partial hydrodynamic equations based on universal hyperbolic equations of the conservation laws:

$$
\frac{\partial U}{\partial t}+\nabla \cdot F(U)=S^{v}(\vec{r})
$$

$U$ - universal vector conserved hydrodynamic quantities such as energy, momenta, net charge densities. $F(U)$ is the flux vector and $S^{v}(\vec{r})$ sources term of modification in the hydrodynamics quantities.

\subsection{Parallel computing on GPU}

The code is object-oriented, written in the $\mathrm{C}++$ language. One of its main features is the use of parallel computing on general-purpose graphical processing units (GPGPU). The objective was able to generate event-by-event heavy-ion collision data in high resolution of the computational grid up to $200^{3}$ in the Cartesian system [5]. We implemented WENO (5th and 7th order) and Runge-Kutta (3rd order) algorithms [6]. For employing graphics card processors Graphics Processing Unit (GPU), we use nVidia CUDA architectures. The algorithms are written in a modular, parallel way and are easy to modify thanks to CUDA C++ features. Out of several memory types available on the GPU we chose surface memory interface for best performance and simple extensibility [7].

\section{Results of simulations $A+A$ interactions}

Initial-state fluctuations can be obtained using Glauber Monte-Carlo, models like UrQMD [8], other string models and another approach. The UrQMD is used to simulate only the early phase of the collision. The time of the UrQMD simulations was set to $1 \mathrm{fm}$.

To evaluate how initial-state fluctuations affect energy density distributions the 100 events $\mathrm{Au}+\mathrm{Au}$ collisions at $200 \mathrm{GeV}$ 0-10\% most central, were generated as initial conditions. Then hydrodynamic simulations with three initial conditions scenario where performed averaging over 1,10, 100 events (Figures 1 and 2). It was studied the deviation of energy density distribution between the initial conditions generated from a single event and an average of 100 events along the OX axis for $\mathrm{y}$ and $\mathrm{z}$ in the center of the system. The Equations 2 ware used to evaluate the discrepancy between the simulation of a single event and the simulation where the initial condition was averaged over 100 events.

$$
\Delta=\sum_{i=0}^{N}\left|A_{i}-S_{i}\right| \quad \Sigma_{E}=\sum_{i=0}^{N} A_{i} \quad \Delta=\frac{\Delta}{\Sigma_{E}} \times 100 \%
$$

$\Delta$ - divergence factor, $\Sigma_{E}$ - the sum of energy density for average conditions, $A_{i}$ - value from the average conditions, $S_{i}$ - value from a single collision, $N$ - number of cells compared 

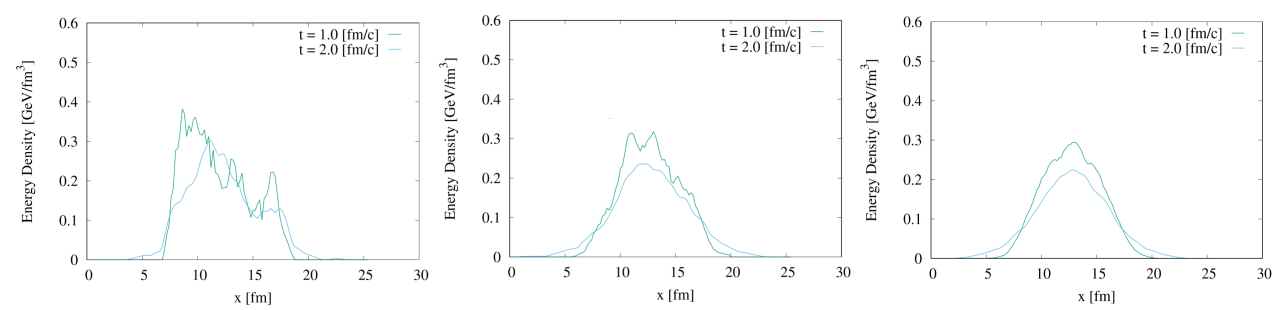

Figure 1. Energy density projection in $1 \mathrm{D}(\mathrm{y}=\mathrm{z}=12.8 \mathrm{fm})$
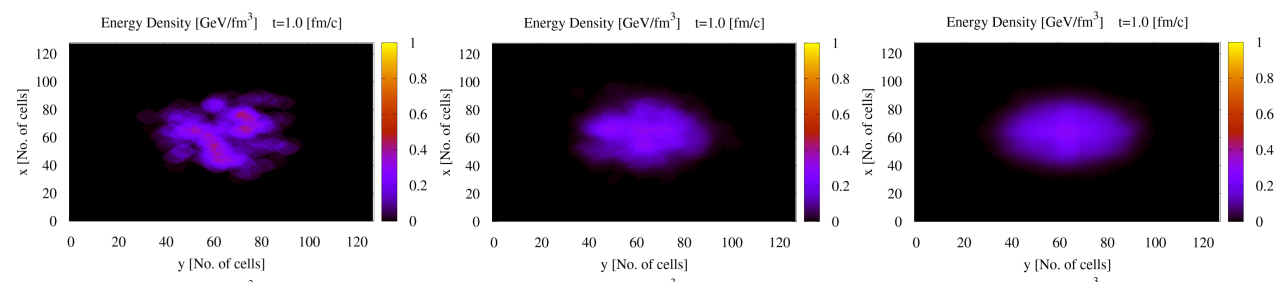

Energy Density $\left[\mathrm{GeV} / \mathrm{fm}^{3}\right] \quad \mathrm{t}=2.0[\mathrm{fm} / \mathrm{c}]$
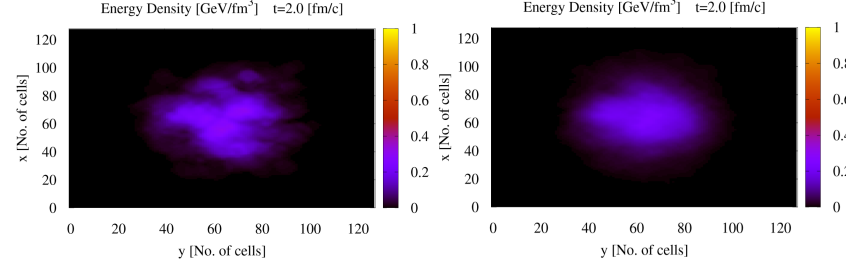

Energy Density $\left[\mathrm{GeV} / \mathrm{fm}^{3}\right] \quad \mathrm{t}=2.0[\mathrm{fm} / \mathrm{c}]$

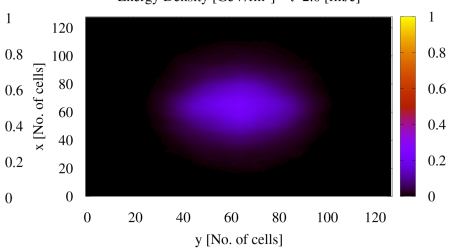

Figure 2. Energy density cross-section in $2 \mathrm{D}$ (xy plane with $\mathrm{z}=12.8 \mathrm{fm}$ )

Table 1. A measure of the discrepancy in the energy density distribution between the conditions generated from single event and averaging of 100 event along the $\mathrm{x}$ axis for $\mathrm{y}=\mathrm{z}=12.8 \mathrm{fm}$.

\begin{tabular}{lllll}
\hline Time $[\mathrm{fm} / \mathrm{c}]$ & 1.5 & 2.0 & 2.5 & 3.0 \\
\hline$\Delta[\%]$ & 33.77 & 27.76 & 25.19 & 28.39 \\
$\Delta\left[\mathrm{GeV} / \mathrm{fm}^{3}\right]$ & 2.13 & 1.18 & 0.66 & 0.44 \\
$\Sigma\left[\mathrm{GeV} / \mathrm{fm}^{3}\right]$ & 6.50 & 4.25 & 2.62 & 1.55 \\
\hline
\end{tabular}




\section{Conclusions}

The energy density distributions were analysed in the three initial condition scenarios (averaging 1,10 and 100 events). The conservation of the initial-state fluctuations was assessed. It has been shown that the energy density discrepancy between the conditions generated from a single event and averaging of 100 event after $1.5 \mathrm{fm}$ is $33.77 \%$ and decreases to $28.39 \%$ during the expansion of matter to $3.0 \mathrm{fm}$. It was also noted that along with the average initial conditions after more collisions ( $>10$ events) energy density distributions begin to resemble an ellipsoid shape. These results confirm the hypothesis of hydrodynamic simulation retains information on fluctuations from initial conditions. Nevertheless, fluctuations decrease as a function of time. Further investigation of the impact of initial-state fluctuations to final particle distributions requires an algorithm of freeze-out to be implemented.

\section{References}

[1] Sikorski, Jan, et al., Journal of Physics: Conference Series. Vol. 509. No. 1. IOP Publishing, (2014)

[2] Tachibana, Yasuki, and Tetsufumi Hirano. Physical Review C 90.2021902 (2014)

[3] Slodkowski Marcin, et al., Journal of Physics: Conference Series (Vol. 1085, No. 5, p. 052001 (2018)

[4] Voloshin, Sergei A., Arthur M. Poskanzer, and Raimond Snellings. Relativistic Heavy Ion Physics. Springer, Berlin, Heidelberg, 293-333 (2010)

[5] Slodkowski Marcin, et al., Federated Conference on Computer Science and Information Systems (pp. 441-446) (2013)

[6] Slodkowski Marcin, et al., International Journal of Nonlinear Sciences and Numerical Simulation, 19(1), 25-35 (2018)

[7] Cygert Sebastian, et al. Concurrency and Computation: Practice and Experience, 27(6), 1591-1602. (2015)

[8] Bass, Steffen A., et al. Progress in Particle and Nuclear Physics 41: 255-369 (1998). 